\title{
The efficacy of transcutaneous electrical nerve stimulation therapy in pain control after cesarean section delivery associated with uterine contractions and abdominal incision
}

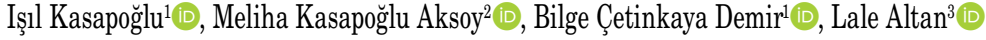 \\ ${ }^{1}$ Department of Obstetrics and Gynecology, Uludağ University School of Medicine, Bursa, Turkey \\ ${ }^{2}$ Department of Physical Medicine and Rehabilitation, University of Health Sciences, Bursa Yüksek Ihtisas Training and Research Hospital, Bursa, Turkey \\ ${ }^{3}$ Department of Physical Medicine and Rehabilitation, Uludağ University School of Medicine, Bursa, Turkey
}

Received: June 08, 2018 Accepted: February 13, 2019 Published online: May 18, 2020

\begin{abstract}
Objectives: This study aims to investigate the effects of transcutaneous electrical nerve stimulation (TENS) therapy on the intensity of pain associated with uterine contractions and abdominal incision in patients undergoing cesarean section (C-section) delivery.

Patients and methods: This single-blind, prospective, randomized-controlled study included a total of 90 female patients (mean age 30.5 years; range, 25 to 36 years) who had a scheduled C-section delivery between November 2017 and April 2018 . Forty-five postpartum patients were randomly assigned into the treatment group (Group 1) and TENS electrodes were placed below and above the abdominal incision. The control group $(n=45)$ consisted of 45 patients who had a scheduled C-section (Group 2) ( $n=45)$ and received routine follow-up care. The Visual Numeric Scale (VNS) scores were obtained separately for abdominal, low back, and groin pain at baseline (within $1 \mathrm{~h}$ after $\mathrm{C}$-section before the TENS replacement), at postpartum 2, 6, 24, and $48 \mathrm{~h}$. In addition to instant scoring, the percentages of change in pain scores from baseline were also calculated for each time points.

Results: Data of a total of 87 patients were analyzed. No statistically significant difference was found in the baseline VNS scores measured in any body regions between the groups ( $>0.05$ ). However, there was a statistically significant difference in the instant VNS scores for abdominal pain at $2,6,24$, and $48 \mathrm{~h}$ in favor of Group $1(\mathrm{p}<0.05)$. No significant differences were found in the instant VNS scores for low back and groin pain $(\mathrm{p}>0.05)$. The comparison of changes in pain scores from baseline (\% change) over time between the groups revealed a statistically significant difference in favor of Group 1 in all VNS scores for abdominal, low back, and groin pain at 2, 6, 24, and 48 $\mathrm{h}(\mathrm{p}<0.05)$. Conclusion: Our study results suggest that TENS is an effective and safe non-invasive, non-pharmacological treatment modality. It may be preferred as an alternative method in pain control in postpartum women after C-section delivery.
\end{abstract}

Keywords: Analgesia, cesarean section, pain control, transcutaneous electrical nerve stimulation.

Cesarean section (C-section) is among the procedures which may cause pain after surgery. ${ }^{[1]}$ Although the intensity of pain varies widely from person to person, a study in women undergoing C-section reported that pain experienced was more intense during the first $24 \mathrm{~h}$ after C-section. ${ }^{[2]}$ Central sensitization induced by the skin incision and tissue damage may result in a more intense and long-term pain. Therefore, the risk for sensitization is associated with intensified postoperative pain and an increased need for analgesics. ${ }^{[3,4]}$ Appropriate and adequate pain management during this period makes it easier to breath, cough, and ambulate for patients and, consequently, it may decrease the prevalence and medical treatment costs of pulmonary, cardiac, and thromboembolic complications. ${ }^{[5]}$

Analgesics to be used to manage acute pain after C-sections are more specific than those which are used after other surgical procedures. Opioids are the most preferred agents in surgical procedures with similar skin incisions in the lower abdominal regions, while the use opioids is limited after C-sections, since these agents may sedate the mother and prevent

Corresponding author: Meliha Kasapoğlu Aksoy, MD. SBÜ Bursa Yüksek İhtisas Eğitim ve Araştırma Hastanesi Fizik Tedavi ve Rehabilitasyon Kliniği, 16800 Ylldırım, Bursa, Türkiye. e-mail: melihakasapoglu@hotmail.com 
her to breastfeed and, additionally, they are secreted into milk and may lead to fatigue and depression in the newborn. ${ }^{[6]}$ Therefore, non-opioid analgesics are preferred in the management of pain after C-sections. ${ }^{[7]}$ However, since non-opioid analgesics are also secreted into milk and may affect the newborn's health, the use of non-pharmacological interventions is important. These interventions may allow avoiding adverse effects associated with pharmacological agents and harm caused by drug-drug interactions. Nonpharmacological interventions to be used for these purposes include massage, hot-packs, hypnotherapy, acupuncture, and transcutaneous electrical nerve stimulation (TENS). ${ }^{[2,6,8]}$

The TENS consists of electrical current with different frequencies delivered through the skin surface electrodes. It is a non-invasive, safe, and low-cost treatment modality to send electrical impulses through the skin and there are reports indicating that TENS can be used as an adjuvant therapy in addition to pharmacological agents. Although the mechanism of action of TENS has remained controversial, its efficacy has been interpreted by the gate-control theory and activation of the endogenous opioid system. ${ }^{[8]}$

The use of TENS in pain relief is effective and simple in postpartum women; however, there is still limited high-level evidence regarding its effectiveness during postpartum period. ${ }^{[8]}$ In the present study, we aimed to investigate the effects of TENS on the intensity of pain associated with uterine contractions and abdominal incision at different time points and to evaluate its effect on a decrease in the amount of additional drug consumption after the C-section delivery.

\section{PATIENTS AND METHODS}

This single-blind, prospective, randomizedcontrolled study included a total of 90 female patients (mean age 30.5 years; range, 25 to 36 years) who had a scheduled C-section delivery at the Obstetrics and Gynecology Clinic of Uludağ University Faculty of Medicine between November 2017 and April 2018. All patients were unable to get into active labor due to various reasons (i.e., prior C-section, prior uterine surgery, acute fetal distress, cephalopelvic disproportion, malpresentation or elective C-section) and underwent a scheduled C-section with a Pfannenstiel incision under general anesthesia. Exclusion criteria were as follows: having any complications, C-section with a median skin incision, C-section under spinal or epidural anesthesia, receiving patient-controlled anesthesia, having a cardiac pacemaker, morbid obesity, epilepsy, preeclampsia/eclampsia, and unwillingness to participate in the study. A written informed consent was obtained from each participant. The study protocol was approved by the Uludağ University Faculty of Medicine Ethics Committee. The study was conducted in accordance with the principles of the Declaration of Helsinki.

The patients were divided into two groups using a computer-generated random table via block randomization with three patients in per block. The random number table was produced with the Random Integer Generator procedure from the following website: http://www.random.org/. A total of 45 postpartum patients were included in the treatment group (Group 1) and TENS (BTL-4000 Smart, 2014; BTL Industries Ltd., Hertfordshire, United Kingdom) electrodes were placed below and above the abdominal incision, and electrical stimulation was applied at $100 \mathrm{~Hz}$ frequency with a pulse width of $75 \mu$ s, for 30-min sessions a day, at the same time every day, during two-day hospital stay. The first session took place within the first postpartum hour, as soon as possible after the $\mathrm{C}$-section procedure was terminated. To achieve maximum relief, the amplitude was gradually increased from 10 to $30 \mathrm{~mA}$, as long as the patient could tolerate it. Before the procedure, the patients were informed about tingling which would occur. The control group (Group 2) included a total of 45 patients who received routine follow-up care.

Patients in both groups received standard analgesic medications (diclofenac sodium/paracetamol). Additional doses of analgesics were administered, if the Visual Numeric Scale (VNS) pain score was $>3$ at any site during the study period, while unnecessary doses were avoided, and total diclofenac sodium and paracetamol doses administered to patients during follow-up (up to $48 \mathrm{~h}$ after surgery) were recorded.

Demographic and clinical characteristics of both groups were recorded and the body mass indices $\left(\mathrm{BMI}, \mathrm{kg} / \mathrm{m}^{2}\right.$ ) were calculated. Gravidity and parity were also recorded. All data were collected with faceto-face interviews by an investigator who was blinded to group allocation.

The VNS was used to measure and monitor the intensity of pain. The VNS is a line marked with numbers 0 to 10 and scores range from 0 to 10 $(0=$ no pain and $10=$ worst pain ever $)$. Patients are asked to select a number that reflects the intensity of their pain. ${ }^{[9]}$ The VNS scores were obtained separately 


\begin{tabular}{|c|c|c|c|c|c|c|c|}
\hline \multicolumn{8}{|c|}{$\begin{array}{l}\text { TABLE } 1 \\
\text { Demographic and clinical characteristics of study population }\end{array}$} \\
\hline & \multicolumn{3}{|c|}{ Group 1 (TENS) $(\mathrm{n}=45$} & \multicolumn{3}{|c|}{ Group 2 (Control) $(\mathrm{n}=42)$} & \multirow[b]{2}{*}{$p$} \\
\hline & Mean \pm SD & Median & Min-Max & Mean \pm SD & Median & Min-Max & \\
\hline Age (year) & $30.6 \pm 4.0$ & & & $30.6 \pm 5.5$ & & & 0.978 \\
\hline Body mass index $\left(\mathrm{kg} / \mathrm{m}^{2}\right)$ & $30.2 \pm 3.5$ & & & $29.2 \pm 3.9$ & & & 0.207 \\
\hline Gravidity & & 3 & $1-5$ & & 2 & $1-7$ & 0.550 \\
\hline Parity & & 2 & $1-5$ & & 2 & $1-6$ & 0.749 \\
\hline Initial abdominal VNS & & 7 & $3-10$ & & 7 & $0-10$ & 0.747 \\
\hline Initial low back VNS & & 6 & $0-10$ & & 5 & $0-10$ & 0.180 \\
\hline Initial groin VNS & & 6 & $0-10$ & & 5 & $0-10$ & 0.208 \\
\hline
\end{tabular}

for abdominal, low back, and groin pain at baseline (as soon as possible after the C-section, before starting TENS), at postpartum $2,6,24$, and 48 h by a single physician and the results were recorded. In addition to instant pain score measurements at predefined time points, the percentages of change (\% change) in pain from baseline to predefined time points were also calculated for all sites, as the pain scores measured at these time points were the variables dependent to baseline pain scores (last pain score/first pain score $\times 100)$.

Time of mobilization after the C-section was recorded and the patients were examined by the same physician to detect any possible hyperemia, any possible discomfort, or side effects where the TENS electrodes were placed. Calibration of TENS device was carried out regularly.

\section{Statistical analysis}

A post-hoc power analysis was performed using a wide impact size based on the findings of the present study. A significant $(\mathrm{d}=0.90)$ effect size was obtained by comparing abdominal VNS scores at $2 \mathrm{~h}$ between the groups. The power obtained using this effect size was found to be 0.90 , when the number of units in the groups was considered.

Statistical analysis was performed using the IBM SPSS for Windows version 24.0 software (IBM Corp., Armonk, NY, USA). Descriptive data were expressed in mean \pm standard deviation (SD) or median (min-max)

\begin{tabular}{|c|c|c|c|c|c|c|c|c|}
\hline \multicolumn{9}{|c|}{$\begin{array}{c}\text { TABLE } 2 \\
\text { nt Visual Numeric Sca }\end{array}$} \\
\hline & \multicolumn{2}{|c|}{ VNS at hour 2} & \multicolumn{2}{|c|}{ VNS at hour 6} & \multicolumn{2}{|c|}{ VNS at hour 24} & \multicolumn{2}{|c|}{ VNS at hour 48} \\
\hline & Median & Min-Max & Median & Min-Max & Median & Min-Max & Median & Min-Max \\
\hline \multicolumn{9}{|l|}{ Abdominal } \\
\hline Group 1 & 2 & $0-7$ & 0 & $0-6$ & 0 & $0-5$ & 0 & $0-2$ \\
\hline Group 2 & 5 & $0-9$ & 4 & $0-10$ & 2 & $0-10$ & 0 & $0-10$ \\
\hline$p$ value & \multicolumn{2}{|c|}{0.002} & \multicolumn{2}{|c|}{$<0.001$} & \multicolumn{2}{|c|}{0.001} & \multicolumn{2}{|c|}{$<0.001$} \\
\hline \multicolumn{9}{|l|}{ Low back } \\
\hline Group 1 & 2 & $0-7$ & 0 & $0-5$ & 0 & $0-4$ & 0 & $0-3$ \\
\hline Group 2 & 4 & $0-10$ & 1 & $0-10$ & 0 & $0-10$ & 0 & $0-8$ \\
\hline$p$ value & \multicolumn{2}{|c|}{0.135} & \multicolumn{2}{|c|}{0.320} & \multicolumn{2}{|c|}{0.498} & \multicolumn{2}{|c|}{0.204} \\
\hline \multicolumn{9}{|l|}{ Groin } \\
\hline Group 1 & 2 & $0-9$ & 0 & $0-6$ & 0 & $0-5$ & 0 & $0-2$ \\
\hline Group 2 & 1.5 & $0-10$ & 0 & $0-10$ & 0 & $0-10$ & 0 & $0-10$ \\
\hline$p$ value & \multicolumn{2}{|c|}{0.506} & \multicolumn{2}{|c|}{0.425} & \multicolumn{2}{|c|}{0.367} & \multicolumn{2}{|c|}{0.204} \\
\hline
\end{tabular}




\begin{tabular}{|c|c|c|c|c|c|c|c|c|}
\hline \multicolumn{9}{|c|}{$\begin{array}{l}\text { TABLE } 3 \\
\text { eline in Visua }\end{array}$} \\
\hline & \multicolumn{2}{|c|}{$\begin{array}{c}\% \text { Change from hour } \\
0 \text { to hour } 2 \text { in VNS } \\
\text { scores } \\
0-2\end{array}$} & \multicolumn{2}{|c|}{$\begin{array}{l}\text { \% Change from } \\
\text { hour } 0 \text { to hour } 6 \text { in } \\
\text { VNS scores } \\
0-6\end{array}$} & \multicolumn{2}{|c|}{$\begin{array}{c}\% \text { Change from } \\
\text { hour } 0 \text { to hour } 24 \text { in } \\
\text { VNS scores } \\
0-24\end{array}$} & \multicolumn{2}{|c|}{$\begin{array}{c}\text { \% Change from } \\
\text { hour } 0 \text { to hour } 48 \text { in } \\
\text { VNS scores } \\
0-48\end{array}$} \\
\hline & Median & Min-Max & Median & Min-Max & Median & Min-Max & Median & Min-Max \\
\hline \multicolumn{9}{|l|}{ Abdominal } \\
\hline Group 1 & -4 & $-8-0$ & -5 & $-10--1$ & -6 & $10--3$ & -7 & $-10-3$ \\
\hline Group 2 & -2 & $-7-7$ & -3 & $-9-10$ & -5 & $-8-7$ & -5.5 & $-10-6$ \\
\hline$p$ value & \multicolumn{2}{|c|}{0.001} & \multicolumn{2}{|c|}{0.001} & \multicolumn{2}{|c|}{0.011} & \multicolumn{2}{|c|}{0.019} \\
\hline \multicolumn{9}{|l|}{ Low back } \\
\hline Group 1 & -4 & $-7-5$ & -5 & $-8-4$ & -5 & $-9-2$ & -6 & $-9-0$ \\
\hline Group 2 & 0 & $-6-5$ & -1.5 & $-8-5$ & -3.5 & $-10-5$ & -4 & $-10-4$ \\
\hline$p$ value & \multicolumn{2}{|c|}{$<0.001$} & \multicolumn{2}{|c|}{0.004} & \multicolumn{2}{|c|}{0.015} & \multicolumn{2}{|c|}{0.034} \\
\hline \multicolumn{9}{|l|}{ Groin } \\
\hline Group 1 & -4 & $-7-5$ & -5 & $-8-4$ & -5 & $-8-2$ & -6 & $-9-0$ \\
\hline Group 2 & -1 & $-7-3$ & -2 & $-8-5$ & -2.5 & $-9-5$ & -3.5 & $-10-5$ \\
\hline$p$ value & \multicolumn{2}{|c|}{0.008} & \multicolumn{2}{|c|}{0.001} & \multicolumn{2}{|c|}{0.005} & \multicolumn{2}{|c|}{0.010} \\
\hline
\end{tabular}

values. For continuous variables, the independent samples t-test (Student's t-test) or the Mann-Whitney U test was used for intergroup comparisons of normally distributed and non-normally distributed variables, respectively. The Fisher's exact test was used for the comparisons of categorical variables. The Friedman's non-parametric test was used to compare paired samples. A two-tailed $p$ value of $<0.05$ was considered statistically significant.

\section{RESULTS}

Three patients from the control group were excluded from the study due to the need for narcotic analgesics. Finally, a total of 87 patients including 45 patients from the TENS group (Group 1) and 42 patients from the control group (Group 2) completed the study. There was no significant difference between the two groups in terms of demographic characteristics, gravidity and parity, and baseline instant VNS scores for abdominal, low back, and groin pain ( $>0.05)$ (Table 1). All patients became ambulatory on postoperative Day 1 . In both groups, there was a significant decrease in pain scores for all three regions over time $(\mathrm{p}<0.001$ and $\mathrm{p}<0.001$ for Group 1 and Group 2, respectively).

However, we observed a statistically significant difference in the abdominal instant pain VNS scores at $2,6,24$, and $48 \mathrm{~h}$ in favor of Group $1(\mathrm{p}<0.05)$. On the other hand, there was no significant difference in the instant VNS scores for low back pain or groin pain ( $\mathrm{p}>0.05)$ (Table 2).

The comparison of changes in pain scores from baseline (\% change) over time between the groups revealed a statistically significant difference in favor of Group 1 in all VNS scores for abdominal, low back, and groin pain at 2, 6, 24, and $48 \mathrm{~h}(\mathrm{p}<0.05)$ (Table 3 and Figures 1-3).

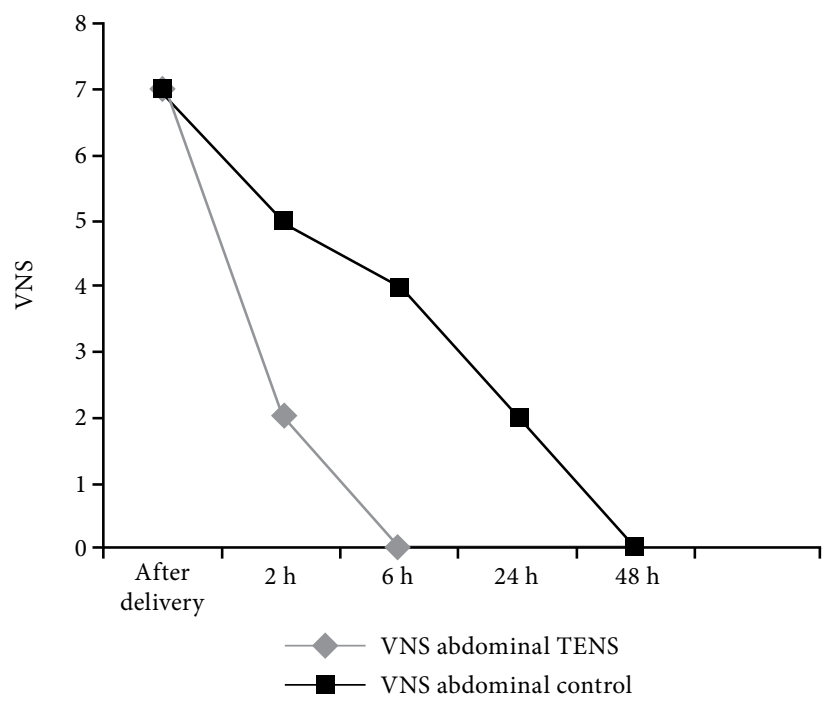

Figure 1. Change from baseline in VNS scores for abdominal pain.

VNS: Visual numeric scale; TENS: Transcutaneous electrical nerve stimulation. 


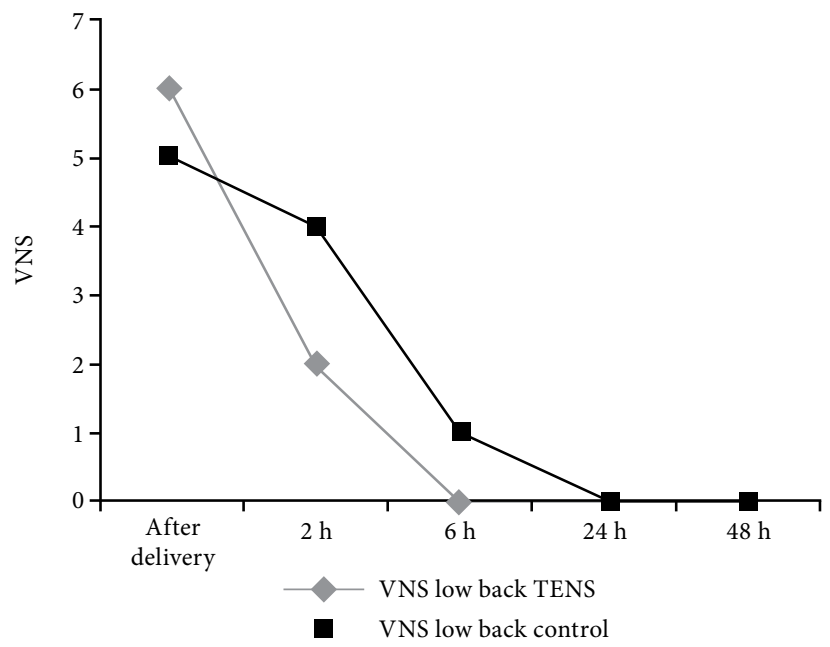

Figure 2. Change from baseline in VNS scores for low back pain.

VNS: Visual Numeric Scale; TENS: Transcutaneous electrical nerve stimulation.

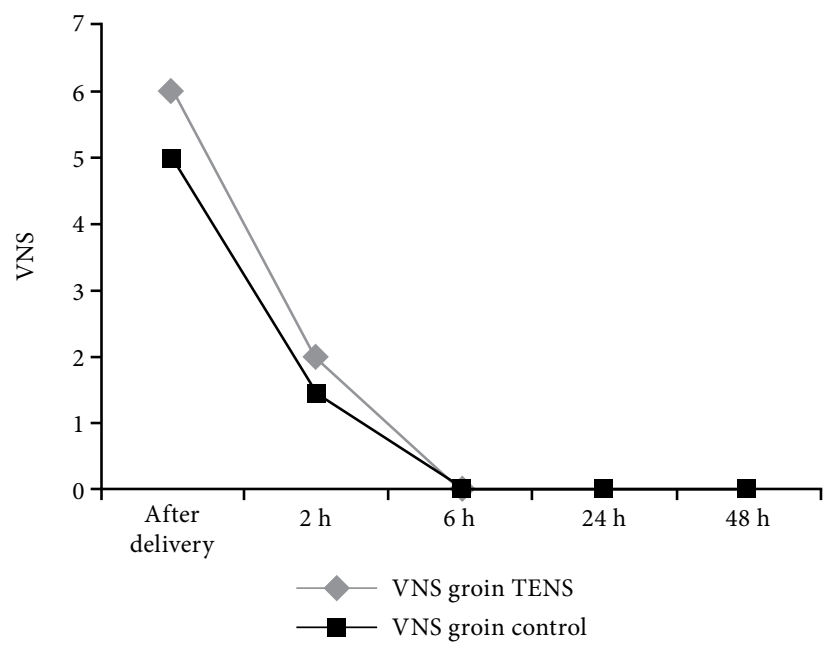

Figure 3. Change from baseline in VNS scores for groin pain. VNS: Visual Numeric Scale; TENS: Transcutaneous electrical nerve stimulation.

There was no significant difference in the amount of additional paracetamol or diclofenac sodium between the groups ( $p>0.05)$ (Table 4). None of the patients developed TENS-related adverse effects.

\section{DISCUSSION}

In our study, we examined the effects of TENS therapy on the intensity of pain associated with uterine contractions and abdominal incision in patients undergoing C-section delivery. Our study results showed that TENS was effective and safe in the management of pain after $\mathrm{C}$-section procedures. In addition, none of the patients who received TENS therapy required narcotic analgesics.

\begin{tabular}{|c|c|c|c|c|}
\hline \multicolumn{5}{|c|}{$\begin{array}{c}\text { TABLE } 4 \\
\text { Comparison of the amount of analgesics }\end{array}$} \\
\hline & \multicolumn{2}{|c|}{ Paracetamol (gr) } & \multicolumn{2}{|c|}{ Diclofenac sodium (mg) } \\
\hline & Median & Min-Max & Median & Min-Max \\
\hline Group 1 & 4 & $0-6$ & 300 & $0-600$ \\
\hline Group 2 & 6 & $2-8$ & 150 & $0-450$ \\
\hline$p$ value & \multicolumn{2}{|c|}{0.156} & \multicolumn{2}{|c|}{0.146} \\
\hline
\end{tabular}

It has been well established that TENS is a noninvasive, safe treatment modality which has been long used in the management of pain. ${ }^{[10]}$ The mechanism of action of TENS has been explained by the gate-control theory and its effects on the activated endogenous opioid system. Originally, TENS was developed and introduced in pain management, once the gate-control theory was defined by Melzack and Wall. ${ }^{[11]}$ According to this theory, the stimulation of large A-beta primary sensory afferent neurons activates inhibitor interneurons in the gelatinous substance of the posterior horns of the spinal cord, resulting in a reduced conduction of nociceptive signals which are conducted through small A-delta and C fibers. ${ }^{[12]}$ In addition, high-frequency TENS exerts its effects on endogenous opioid system by activating delta opioid receptors and low-frequency TENS exerts its analgesic effects on endogenous opioid system by activating mu-opioid receptors and increasing the endorphin levels in blood and cerebrospinal fluid..$^{[8,10,11]}$

Limited studies in the literature have evaluated TENS for postoperative care after C-section. Smith et al. ${ }^{[13]}$ found that TENS was more effective in relieving superficial (skin) pain and pain on movement after a C-section, compared to placebo TENS, while there was no significant difference in reducing the need for analgesics between TENS and placebo TENS. In line with the results reported by Smith et al., ${ }^{[13]}$ TENS was found to be ineffective in reducing the need for analgesics after $\mathrm{C}$-section by Reynold et al. ${ }^{[14]}$ However, Nuñez and Carrasco ${ }^{[15]}$ found TENS to be effective in pain control during earlier periods after the $\mathrm{C}$-section with a $50 \%$ reduction in analgesic consumption. In our study, patients in both groups received routine analgesic medications at baseline (diclofenac sodium/ paracetamol) to avoid any interruption of standard postoperative care protocols for C-section patients. With similar total analgesic doses, TENS group had significantly lower VNS scores for abdominal pain along with improved pain relief for all body parts evaluated in this study. Although the patients in both groups received similar analgesic doses, the fact that 
the instant pain scores at different time points in the postoperative period were found to be significantly lower in the TENS group only in the abdominal region can be explained by the placement localization of the TENS electrodes.

A meta-analysis on the use of TENS in the management of postoperative pain in patients who underwent various surgical procedures (i.e., abdominal, thoracic or lumbar), including a total of 21 randomizedcontrolled studies which compared TENS with placebo in 1,350 patients concluded that the need for analgesia was reduced by $35 \%$ in patients who received TENS, compared to those who received placebo ${ }^{[8]}$ In addition, 50 of 1,350 patients in this meta-analysis were from two separate studies conducted in postpartum woman who received TENS therapy in the management of pain after C-section. In the first study, TENS was found to be $44 \%$ more effective than placebo in 18 patients, while TENS did not make any contribution to epidural anesthesia in the remaining 32 patients. ${ }^{[13,16]}$ Therefore, patients who received epidural anesthesia were not included in our study. In another metaanalysis including patients who underwent thoracic surgery, the addition of TENS to pharmacological analgesia provided a more effective pain relief than placebo TENS or pharmacological analgesia alone, consistent with the results of our study. ${ }^{[17]}$

Pain scores at a single time point were measured after the administration of TENS therapy in a randomized-controlled study assessing the use of TENS in the management of postpartum pain in women who gave birth by C-section, and single time-point pain scores were found to be significantly lower in patients who received TENS therapy, compared to those who received placebo. ${ }^{[18]}$ There is a limited number of studies on the use of TENS in the management of postpartum pain and, unlike previous studies, pain scores were measured sequentially at different sites (i.e., abdomen, groin, and low back), at different time points in our study. Furthermore, for the first time, the percentage of changes from baseline in VNS scores as the indicator of pain relief was also assessed. The percentage of changes from baseline in VNS scores significantly favored TENS for all body sites. The long-lasting effect of TENS applied within the first hour by reducing the pain on the low back and groin, could be explained with the placebo effect and the change in frequency of uterine contractions.

Currently, there are different types of TENS used for various purposes and the types of TENS differ from each other in frequency, amplitude, or pulse duration. The most widely used two modes are highfrequency conventional TENS and low-frequency acupuncture-like TENS. The effect of conventional TENS starts earlier than acupuncture-like TENS; however, its analgesic effect is shorter. ${ }^{[19]}$ It has still remained controversial whether the effects of highfrequency TENS and low-frequency TENS differ from each other. ${ }^{[20]}$ In a study, the efficacy of conventional TENS on cold-induced pain was compared with acupuncture-like TENS, and none of them was found to be superior to the other in providing analgesia. ${ }^{[21]} \mathrm{In}$ line with this finding, in a study in knee osteoarthritis, Vance et al. ${ }^{[22]}$ found that both high-frequency TENS and low-frequency TENS reduced pain, while they exerted similar analgesic effects on pain at rest pain and pain on movement. In our study, we preferred to use conventional TENS in all patients for feasibility reasons. In addition, the analgesic effect of the conventional TENS starts earlier than acupuncturelike TENS and the two modes do not differ from each other regarding their analgesic effects. ${ }^{[23]}$

In meta-analyses and reviews of studies evaluating the efficacy of TENS in postoperative pain control, no clear conclusion can be reached regarding the efficacy of TENS due to randomization of studies and heterogeneity of TENS administration. ${ }^{[24]}$ In our study, although we concluded that TENS was effective in the management of pain after C-section, we were unable to find any statistically significant difference between the TENS group and control group in additional drug consumption in patients with a VNS score of $>3$. Routine follow-up time and the least amount of medications needed remained the same for both groups.

The review of systematic meta-analyses reveals that there is insufficient evidence to draw a definitive conclusion on the efficacy of TENS in the management of postpartum pain control. ${ }^{[25]}$ The main cause underlying the lack of adequate and reliable data is the heterogeneity of studies reported in the literature. A standard way or method has not been defined for the use of TENS so far, and there are no reliable homogenous data. Therefore, there is a need for further studies using different frequencies and amplitudes in larger populations.

The main limitations of the present study are the inability to use a placebo TENS group as the control group and a relatively small sample size.

In conclusion, our study results suggest that TENS is an effective and safe non-invasive, 
non-pharmacological treatment modality with a wide range of clinical applicability. It may be preferred as an alternative method in pain control in postpartum women after $\mathrm{C}$-section delivery.

\section{Declaration of conflicting interests}

The authors declared no conflicts of interest with respect to the authorship and/or publication of this article.

\section{Funding}

The authors received no financial support for the research and/or authorship of this article.

\section{REFERENCES}

1. Siddik SM, Aouad MT, Jalbout MI, Rizk LB, Kamar GH, Baraka AS. Diclofenac and/or propacetamol for postoperative pain management after cesarean delivery in patients receiving patient controlled analgesia morphine. Reg Anesth Pain Med 2001;26:310-5.

2. Karlström A, Engström-Olofsson R, Norbergh KG, Sjöling M, Hildingsson I. Postoperative pain after cesarean birth affects breastfeeding and infant care. J Obstet Gynecol Neonatal Nurs 2007;36:430-40.

3. Berkman ND, Thorp JM Jr, Lohr KN, Carey TS, Hartmann KE, Gavin NI, et al. Tocolytic treatment for the management of preterm labor: a review of the evidence. Am J Obstet Gynecol 2003;188:1648-59.

4. Woolf CJ, Thompson SW. The induction and maintenance of central sensitization is dependent on N-methyl-D-aspartic acid receptor activation; implications for the treatment of post-injury pain hypersensitivity states. Pain 1991;44:293-9.

5. Bonnet F, Marret E. Postoperative pain management and outcome after surgery. Best Pract Res Clin Anaesthesiol 2007;21:99-107.

6. Hepner D, Eappen S. Postoperative analgesia: Systemic and local techniques. In: Chestnut DH, Polley LS, Wong CA, Tsen LC, editors. Chestnut's obstetric anesthesia principles and practice. 4th ed. Philadelphia: Mosby; 2009. p. 575-92.

7. Elyasi H, Heidari M, Shahbeigi S. Comparative effects of indomethacin suppository and pethidine on pain after cesarean section. Pajoohandeh J 2000;5:9-15.

8. Bjordal JM, Johnson MI, Ljunggreen AE. Transcutaneous electrical nerve stimulation (TENS) can reduce postoperative analgesic consumption. A meta-analysis with assessment of optimal treatment parameters for postoperative pain. Eur J Pain 2003;7:181-8.

9. Ritter PL, González VM, Laurent DD, Lorig KR. Measurement of pain using the visual numeric scale. J Rheumatol 2006;33:574-80.

10. Hamza MA, White PF, Ahmed HE, Ghoname EA. Effect of the frequency of transcutaneous electrical nerve stimulation on the postoperative opioid analgesic requirement and recovery profile. Anesthesiology 1999;91:1232-8.
11. Wang B, Tang J, White PF, Naruse R, Sloninsky A, Kariger $\mathrm{R}$, et al. Effect of the intensity of transcutaneous acupoint electrical stimulation on the postoperative analgesic requirement. Anesth Analg 1997;85:406-13.

12. Melzack R, Wall PD. Pain mechanisms: a new theory. Science 1965;150:971-9.

13. Smith CM, Guralnick MS, Gelfand MM, Jeans ME. The effects of transcutaneous electrical nerve stimulation on post-cesarean pain. Pain 1986;27:181-93.

14. Reynolds RA, Gladstone N, Ansari AH. Transcutaneous electrical nerve stimulation for reducing narcotic use after cesarean section. J Reprod Med 1987;32:843-6.

15. Navarro Nuñez C, Pacheco Carrasco M. Transcutaneous electric stimulation (TENS) to reduce pain after cesarean section. Ginecol Obstet Mex 2000;68:60-3.

16. Davies JR. Ineffective transcutaneous nerve stimulation following epidural anaesthesia. Anaesthesia 1982;37:453-4.

17. Sbruzzi G, Silveira SA, Silva DV, Coronel CC, Plentz RD. Transcutaneous electrical nerve stimulation after thoracic surgery: systematic review and meta-analysis of 11 randomized trials. Rev Bras Cir Cardiovasc 2012;27:75-87.

18. Kayman-Kose S, Arioz DT, Toktas H, Koken G, Kanat-Pektas $\mathrm{M}$, Kose M, et al. Transcutaneous electrical nerve stimulation (TENS) for pain control after vaginal delivery and cesarean section. J Matern Fetal Neonatal Med 2014;27:1572-5.

19. Johnson M. Transcutaneous electrical nerve stimulation: Mechanisms, clinical application and evidence. Rev Pain 2007;1:7-11.

20. Olsén MF, Elden H, Janson ED, Lilja H, Stener-Victorin E. A comparison of high- versus low-intensity, high-frequency transcutaneous electric nerve stimulation for painful postpartum uterine contractions. Acta Obstet Gynecol Scand 2007;86:310-4.

21. Francis RP, Marchant PR, Johnson MI. Comparison of post-treatment effects of conventional and acupuncturelike transcutaneous electrical nerve stimulation (TENS): A randomised placebo-controlled study using cold-induced pain and healthy human participants. Physiother Theory Pract 2011;27:578-85.

22. Vance CG, Rakel BA, Blodgett NP, DeSantana JM, Amendola A, Zimmerman $\mathrm{MB}$, et al. Effects of transcutaneous electrical nerve stimulation on pain, pain sensitivity, and function in people with knee osteoarthritis: a randomized controlled trial. Phys Ther 2012;92:898-910.

23. Ilhanli I. Conventional, Acupuncture like or brief intense:Is there any difference between TENSE modalities acording to outcomes of choronic low back pain with lomber disc herniation. Clin Med Res 2015;4:143-50.

24. Vance CG, Dailey DL, Rakel BA, Sluka KA. Using TENS for pain control: the state of the evidence. Pain Manag 2014;4:197-209.

25. Jones L, Othman M, Dowswell T, Alfirevic Z, Gates S, Newburn M, et al. Pain management for women in labour: an overview of systematic reviews. Cochrane Database Syst Rev 2012;3:CD009234. 